\title{
Hospitales reformando al mundo verde
}

ISSN 1794-9831

E-ISSN 2322-7028

Vol. 13 No. 2

Jul - Dic 2016

Cúcuta, Colombia

\author{
Sandra Milene Rojas-Criollo*
}

\section{RESUMEN}

Introducción: factores ambientales como el cambio climático, el calentamiento global, el mal uso del suelo y del agua, la mala administración de los recursos naturales, la contaminación del aire en espacios abiertos y cerrados, el crecimiento urbano y la industrialización han ocasionado cambios en los procesos naturales del planeta; para la Organización Mundial de la Salud constituyen una barrera significativa que impide alcanzar los objetivos del milenio y del desarrollo sostenible, haciendo cada vez más grave su aplicación y generando un ambiente hostil para la vida de las personas. Metodología: investigación documental, descriptiva, con enfoque cualitativo. Se realizó búsqueda en las bases de datos Ebsco, Proquest, Scielo, Elsevier, etc., con descriptores en inglés y español. Se clasificó los artículos por similitudes, autores y definiciones; se agruparon 60 artículos en una matriz y, posteriormente, se analizaron y se integraron los conceptos para luego categorizarlos. Conclusiones: es de gran relevancia el conocimiento sobre hospitales verdes, ya que son empresas que promueven la salud pública y la reducción del impacto ambiental, además de la disminución de los índices de morbilidad y mortalidad. El personal de salud tiene un papel importante para desarrollar e implementar estas actividades en beneficio del ambiente y las personas.

PALABRAS CLAVES: contaminación ambiental, medio ambiente, salud ambiental, residuos sanitarios $^{* *}$.

* Enfermera.

Magister en

Educación.

Docente y Asesora

opciones de

grado. Fundación

Universitaria del

Área Andina.

Bogotá D.C.,

Colombia. Correo

electrónico:

srojas@areandina.

edu.co

** Descriptores

en Ciencias de la

Salud (DeCS), en

la página $\mathrm{http}: / /$

decs.bvs.br/E/

homepagee.htm

de la Biblioteca

Virtual en Salud del proyecto BIREME,

de la Organización

Mundial de la

Salud y de la

Organización

Panamericana de la

Salud. 


\section{Hospitals reforming the green world}

Introduction: environmental factors as climate change, global warming, bad usage of the soil and water, the bad administration of the natural resources, air pollution in open and closed spaces, urban growth and industrialization has caused changes on the natural processes of the Earth; for the World Health Organization, these factors are a significant barrier that prevents reaching the millennium development goals and the sustainable development, increasing the difficulty of its application, generating a hostile environment for the life of individuals. Methodology: documentary research, descriptive with qualitative focus. Research was done in these databases: Ebsco, Proquest, Scielo, Elsevier etc., with keywords in English and Spanish. Articles classified by similarity, authors, and definitions; 60 articles were grouped in a matrix, subsequently analyzed and integrated to the concepts, and categorized. Conclusions: Knowledge about green hospitals is of great importance, since these hospitals are companies that promote public health and reduction of environmental impact, also the decrease of mortality and morbidity rate. Health workers have an important role to develop and implement these activities for the benefit of the environment and individuals.

KEYWORDS: environmental pollution, environment, environmental health, medical waste. 


\section{Hospitais para reformar o mundo verde}

\section{RESUMO}

Introdução: fatores ambientais como a mudança climática, o aquecimento global, o mau uso do solo e d'água, a má gestão dos recursos naturais, a poluição do ar em espaços abertos e fechados, o crescimento urbano e a industrialização tem ocasionado mudanças nos processos naturais do planeta; para a Organização Mundial da Saúde, constituem uma barreira significativa que impede alcançar os objetivos do milênio e do desenvolvimento sustentável, fazendo cada vez mais grave sua aplicação, gerando um ambiente hostil para a vida das pessoas. Materiais e Métodos: pesquisa documental, descritiva, com abordagem qualitativa. Realizou-se busca nas bases de dados: Ebsco, Proquest, Scielo, Elsevier etc., com palavras chaves em inglês e espanhol. Ele se classificou os artigos por semelhanças, autores e, definições, e foram agrupados numa matriz 60 artigos; posteriormente, se analisaram e integraram os conceitos em seguida categorizar. Conclusões: é de grande relevância o conhecimento sobre hospitais verdes, já que são empresas que promovem a saúde pública e a redução do impacto ambiental, além da diminuição dos índices de morbidade e mortalidade. O pessoal de saúde tem um papel importante para desenvolver e implementar estas atividades em benefício do ambiente e das pessoas.

PALAVRAS-CHAVE: poluição ambiental, meio ambiente, saúde ambiental, resíduos de serviços de saúde 
E-ISSN 2322-7028

Vol. 13 No. 2

Jul - Dic 2016

Cúcuta, Colombia

\section{INTRODUCCIÓN}

La contaminación se inició a finales del siglo XVIII, cuando se presentó la revolución industrial; la situación se agravó después de la Segunda Guerra Mundial y desde allí el mundo empezó a darse cuenta que hubo un excesivo uso de energía (1).

En la actualidad, el exagerado cambio climático, el crecimiento urbano, la industrialización y el calentamiento global han afectado los procesos naturales del continente y el planeta. Además, las ciudades están cubiertas de cemento, asfalto y otras sustancias que han generado aceleramiento de los ciclos naturales del aire y del agua, alterando los procesos de los ecosistemas; es por esto que el mundo ha tomado conciencia para reestablecer el balance natural del medio ambiente urbano y de las grandes ciudades, al generar nuevamente espacios verdes (áreas cubiertas de vegetación) para mejorar así las condiciones de las ciudades y los habitantes, tratando de equilibrar la temperatura, mejorar el mantenimiento y absorción de las aguas lluvias, fomentar la biodiversidad y mejorar la salud de las personas (2).

Otras actividades como el reciclaje de basuras, el manejo de los residuos hospitalarios en forma apropiada, el fomento de la biodiversidad, la iniciación de hospitales y terrazas buscan proteger el medio ambiente, disminuir los riesgos de la salud de las personas y, por ende, la prevención de la enfermedad (2), cumpliendo de esta manera con los 17 Objetivos para el Desarrollo Sostenible (ODS), dentro de los cuales se cuenta: erradicar la pobreza, combatir la desigualdad, promover la prosperidad, el saneamiento, el consumo de agua limpia y disminuir la contaminación, entre otros, con el propósito de contribuir a la protección del medio ambiente (3).

La contaminación ambiental ${ }^{\dagger}$ existe desde siempre, hace parte del ser humano y de las diferentes actividades que realiza, aumentando la gravedad de los incidentes en la contaminación en el mundo, los cuales han generado alteración en el ambiente y la salud.

\footnotetext{
Se define contaminación ambiental como la presencia en el ambiente de cualquier agente (físico, químico o biológico) o combinación de varios agentes en lugares, formas y concentraciones que pueden ser nocivas para la salud, seguridad o bienestar de la población, o pueden ser perjudiciales para la vida vegetal y animal (4).
}

Como agentes de contaminación, las sustancias naturales o sintéticas afectan el ambiente porque extralimitan la capacidad de los ecosistemas en la asimilación y degradación de los productos, aumentado dicha contaminación. Debe existir un equilibrio del sistema, planeta, organismo, ciudad o río en cualquier forma de materia o energía que entra y debe salir tarde o temprano; si no ocurre, esta materia y energía se acumula en el sistema y ocurre la contaminación, la cual puede ser de origen natural o causada por el hombre (biológica, física, química) (1).

Añádase a esto que los efectos combinados del cambio climático, la contaminación química y el uso no sustentable de los recursos ha permitido que se presente la incidencia de enfermedades en todo el mundo. Además, se han incrementado los problemas de salud ambiental, así que los sistemas de salud han aumentado las exigencias para el manejo de los recursos, ya que este sector, en algún momento, ha contribuido a agravar estos problemas.

Las actividades de renaturalización han permitido el acercamiento entre la naturaleza y los habitantes de la ciudad a través de la organización, promoción e integración de los diferentes ecosistemas, la consolidación y ampliación de la estructura ecológica principal, la construcción de escenarios fundamentales para la conectividad ecológica, la recuperación de humedales, ríos y quebradas, y la participación de la comunidad, de tal manera que se produzca un acercamiento entre la naturaleza y los habitantes de la ciudad (5-6).

Esta promoción y combinación de los diferentes ecosistemas ha permitido que la ciudad se vuelva más verde, a partir del uso de nuevas y diferentes tecnologías como techos y pantallas verdes, muros y mobiliario verde, densificando con árboles y jardinería los espacios verdes existentes y recuperando zonas endurecidas, estimulando la agricultura urbana y los jardines agroecológicos, analizando los diferentes territorios para que los habitantes de la ciudad construyan y usen el paisaje de acuerdo con las necesidades particulares $\mathrm{y}$, también, mejorando la imagen de las ciudades y la salud mental de la comunidad $(5,7)$.

En este proceso, resulta de vital importancia el personal médico y de enfermería, los hospitales, los 
sistemas y el Ministerio de Salud, ya que cumplen un papel cada vez más relevante en estas soluciones y pueden conducir la transformación de sus propias instituciones, además de convertirse en impulsores de políticas y de prácticas que van a promover la salud ambiental y la promoción de la salud pública, ahorrando y mejorando, al mismo tiempo, los recursos financieros.

La finalidad de esta revisión consiste en evidenciar las repercusiones de la contaminación ambiental en la salud humana y la importancia de las terrazas verdes como una de las estrategias ecológicas para contrarrestar sus efectos nocivos. Además, el gobierno, la sociedad, los administradores y el equipo de salud podrán diseñar y aplicar medidas para enfrentar estos cambios, disminuyendo la vulnerabilidad de los territorios rurales, urbanos, ciudades y la población en general.

\section{METODOLOGÍA}

La metodología que se utilizo fue la investigación documental ${ }^{\ddagger}$, descriptiva, con enfoque cualitativo, en aras de exponer la importancia del conocimiento de los hospitales verdes que, por medio de la adopción y orientación de nuevas políticas y normas, favorecen de manera continua la promoción de la salud ambiental en el sector salud y la sociedad.

Se realizó una búsqueda de información relacionada con el tema en artículos publicados durante el periodo 2002 a 2016, en las diferentes bases de datos de información científica Ebsco, base de datos bibliográficos Proquest, el portal bibliográfico Dianelt, biblioteca científica electrónica Scielo, documentos publicados por la editorial Elsevier y el sistema de información académica para América Latina y el Caribe Latindex, entre otros, con palabras claves en inglés y en español como: contaminación ambiental, medio ambiente, salud ambiental y residuos sanitarios.

Se procedió a clasificar los artículos por similitudes, autores y definiciones; se agruparon 60 artículos en

\footnotetext{
\#studio metódico, sistémico y ordenado con objetivos bien definidos, de datos, documentos escritos, fuentes de información impresas, contenidos y referencias bibliográficas, los cuales, una vez recopilados, contextualizados, clasificados, categorizados y analizados, sirven de base para la comprensión del problema, para la construcción del nuevo conocimiento (8).
}

una matriz para, posteriormente, realizar el análisis y la sistematización, actividades en las cuales se integraron los conceptos para luego realizar una descripción de lo investigado por medio de categorías. De los 60 artículos, el 16,66\% (10 artículos) correspondían al idioma inglés y 54 artículos (90\%) se referían a temas como medio ambiente, hospitales verdes y sustancias.

Los criterios de selección de los artículos fueron: literatura de los últimos 10 años, artículos válidos por importancia histórica, artículos en idioma inglés y español, artículos de bases de datos e indexados y artículos de orden nacional e internacional. Se emplearon como criterios de exclusión: artículos de rango de tiempo mayor a 10 años y artículos que no pertenecieran a bases de datos (blogs, periódicos).

\section{En la búsqueda de una cultura verde}

Durante mucho tiempo, la economía capitalista, el socialismo, la globalización y la industrialización han desconocido las bondades de la naturaleza, creando, a partir de ello, una reacción negativa en el manejo inadecuado de los recursos naturales renovables y no renovables, desatando así un deterioro en el ambiente, tal como lo menciona Molina (9): "Durante demasiado tiempo la industrialización mundial junto a la economía capitalista y el socialismo, ha ignorado las leyes de la naturaleza". Por esta razón, se empezó a recapacitar y pensar en el manejo adecuado de esos sistemas de producción, como en el caso de los hospitales.

En la primera mitad del siglo XXI, para la Organización Mundial de la Salud (OMS), los factores ambientales como el agua no apta para su consumo, las malas condiciones sanitarias y de higiene, la contaminación del aire en espacios abiertos y cerrados, los riesgos en el lugar de trabajo, los accidentes industriales, los accidentes automovilísticos, el cambio climático, el mal uso del suelo y la mala administración de los recursos naturales constituyen barreras para el logro de los objetivos del Milenio, ya que aproximadamente una cuarta parte de las enfermedades y muertes que se producen en el mundo son atribuibles a estos factores $(2,10)$.

A partir de lo anterior, se han creado mecanismos de gestión para el mejoramiento de este fenómeno en

.


E-ISSN 2322-7028

Vol. 13 No. 2

Jul - Dic 2016

Cúcuta, Colombia muchos niveles, los cuales han impulsado el cambio generando resultados para los grupos humanos y disminuyendo los peligros (2).

\section{Los riesgos ambientales en los hospitales}

Se entiende por salud ambiental "el área de las ciencias que trata la interacción y los efectos que, para la salud humana, representa el medio en el que habitan las personas" (9), siendo componente esencial de salud pública que promueve la calidad de vida. La Organización Mundial de la Salud (OMS) plantea que "la salud ambiental comprende aquellos aspectos de la salud humana, incluyendo la calidad de vida, que son determinados por factores físicos, químicos, biológicos, sociales y psicológicos en el medio ambiente" (11-12).

La salud ambiental se puede entender como aquello relacionado con todos los factores físicos, químicos y biológicos externos e internos de una persona, abarcando factores que inciden en la salud, en la prevención de la enfermedad y en la conformación de entornos favorables para la salud y el ambiente (13).

La Organización Mundial de la Salud, desde una perspectiva global, muestra los diferentes riesgos ambientales atribuibles a morbilidad que son consecuencia de factores ambientales, enfatizando en enfermedades como la diarrea, las infecciones de las vías respiratorias inferiores, distintos tipos de lesiones accidentales y el paludismo. Además, destaca que la morbilidad causada por factores ambientales es mucho más elevada en los países en vía de desarrollo, pues en ellos se incrementa la incidencia de enfermedades no transmisibles como las enfermedades cardiovasculares y los cánceres (13).

Adicionalmente, se menciona que la población infantil es la más afectada por las enfermedades provocadas por factores ambientales, que cobran cada año la vida de más de cuatro millones de niños, principalmente en estos países en desarrollo. En cuanto a la tasa de mortalidad del recién nacido por causa de estos factores, se informa que es 12 veces mayor en los países en desarrollo que en los países desarrollados (13). Se calcula que el $24 \%$ de la carga de morbilidad mundial y el $23 \%$ de todos los fallecimientos puede atribuirse a factores ambientales. Por ello, es necesario trabajar en la búsqueda de los ambientes saludables, con el ánimo de disminuir esta mortalidad y mejorar la salud humana e infantil (13).

Por otro lado, los hospitales son empresas que proporcionan asistencia médico-clínica desempeñando funciones de prevención, de rehabilitación, de formación y de investigación, pero, infortunadamente, al cumplir sus funciones, provocan efectos indeseables y no muy agradables conocidos como contaminación hospitalaria (7).

El sector salud ha generado efectos sobre el ambiente. Por ejemplo, el Servicio Nacional de Salud de Inglaterra "ha calculado que su huella de carbono es de más de 18 millones de toneladas de $\mathrm{CO} 2$ por año, siendo el $25 \%$ de las emisiones del sector público. En Brasil, los hospitales utilizan enormes cantidades de energía que representa más del $10 \%$ del total del consumo energético comercial del país. En Estados Unidos, el sector de la salud es el principal usuario de sustancias químicas, con un conocido efecto cancerígeno. En China, el gasto del sector de la salud en construcciones supera los 10 mil millones de dólares por año" (7).

Por consiguiente, los residuos médicos patogénicos que son arrojados detrás de una clínica rural o urbana como resultado de una campaña de vacunación o de residuos hospitalarios, además de la contaminación del aire generada por el alto consumo de energía de un hospital de tercer nivel, generan daño en la salud ambiental.

En Colombia se mencionan aspectos ambientales que han marcado historia, como el manejo de los residuos sólidos en la prestación de los servicios de aseo (higiénico y sanitario), forjando aumento en las enfermedades (11). Este problema comenzó cuando la comunidad empezó a dejar los residuos en las vías públicas, creando la necesidad de establecer un método de recolección inapropiado por parte del servicio público de Bogotá, disponiendo los residuos en descarga a cielo abierto en un 56,19\%, generando, de esta manera, más contaminación al medio ambiente, el agua y el aire debido a otras actividades conexas como la quema de basuras a cielo abierto y la generación de gases producidos por estas basuras, gases de invernadero y gases degradadores de la capa de ozono, los rellenos sanitarios, etc. (14).

Cuando el manejo y gestión de los residuos sólidos no 
se efectúa adecuadamente, se produce rápidamente contaminación. Por ejemplo, la incineración de residuos genera problemas de contaminación del aire y eliminación de cenizas. Se busca la solución al problema pero, a menudo, al ejecutar el trabajo no se realiza adecuadamente, aumentando los costos (15). El buen manejo de las basuras es decisivo para proteger las fuentes de agua y lograr la sostenibilidad ambiental de las ciudades.

Otros factores asociados al manejo actual dado a los residuos sólidos y que afectan el medio ambiente son, por ejemplo, las estrategias de mercadeo y sistemas de producción insostenibles que inciden en los patrones de consumo, la falta de conciencia ciudadana relacionada con los residuos, el medio ambiente y la economía de la familia y la nación, la ausencia de apoyo de tecnologías limpias e inexistencia de establecimientos responsables de sectores productivos que generan manejo y disposición de residuos $(16,18)$.

Así mismo, la recolección indiscriminada de todo tipo de residuos como los tóxicos y los peligrosos, aumentando el riesgo ambiental. Ejemplo de ello ocurre en Colombia, donde se llevó para disposición final 26.537 toneladas de residuos sólidos, relacionados con el aumento de la tasa de crecimiento de la población en $1,18 \%$, es decir, se ha aumentado la producción de residuos por habitante. Por ello, es importante evaluar el modo de disposición de los residuos para disminuir la contaminación del agua, suelo y aire $(17,19)$.

Cuando los residuos se disponen en basureros a cielo abierto o vertederos no controlados, son fuente de proliferación de vectores de enfermedades tales como insectos y roedores. Lo mismo ocurre, cuando se presenta la contaminación de las aguas subterráneas y las superficiales, la producción de incendios dando lugar a la contaminación atmosférica y a afecciones en el sistema respiratorio de la comunidad expuesta a los humos generados por su combustión (algunas veces por autocombustión, los olores molestos), la presencia de roedores, insectos y animales domésticos, comportándose como agentes portadores de enfermedades (20).

El sector salud ha evidenciado que han aumentado las enfermedades causadas por vectores como moscas, cucarachas, piojos, pulgas y roedores; asimismo, los microorganismos pueden trasmitirse no sólo a través de las partes externas de los vectores sino también a través de otros fluidos como el vómito, las heces o los líquidos de los mismos. Estos vectores llevan y transmiten agentes patógenos como virus, bacterias, hongos y parásitos, generando transmisión de enfermedades. En este contexto, los residuos sólidos mal manejados generan problemas que repercuten en el estado de salud de la comunidad y en el medio ambiente (21-22).

Como problema de salud pública se ha presentado el mal manejo y la no recolección y disposición adecuada de ellos, fenómeno que aumenta la reproducción de ratas, moscas y otros transmisores de enfermedades, además de la contaminación del aire y del agua que han sido relacionados con el almacenamiento, recogida y evacuación de los desechos sólidos, pudiendo ocasionar un aumento en el número de casos de enfermedades como dengue, leptospirosis, distintos trastornos gastrointestinales, dificultades respiratorias e infecciones dérmicas, y se magnifica cuando la población carece de servicios básicos de saneamiento (23).

Se puede indicar que, sin duda, el daño ambiental continúa creciendo en el mundo $\mathrm{y}$, asimismo, continúa el incremento de la contaminación. Por ejemplo: el aire que se respira hoy en día, el agua y los productos que se consumen cada vez más contaminados, la quema de combustibles fósiles que contaminan el aire y aumentan el asma, las afecciones cardiacas, las enfermedades infecciosas en las personas y el calor producido por el cambio climático. Esto ha contribuido a aumentar los problemas que está enfrentando el mundo y que, según informa la Organización Mundial de la Salud, siete millones de personas por año mueren por combustibles fósiles -más muertes que las que generan el síndrome de inmunodeficiencia adquirida (SIDA), la malaria y la tuberculosis-. Aquí el sector salud debe tener un papel protagónico para ayudar a mejorar esta crisis (24-25).

El sector de la salud empezó a darse cuenta de la relación existente entre el aumento de las altas temperaturas que se ha generado en el ambiente y el aumento de los problemas de vías respiratorias agudas y crónicas en los niños y ancianos. Además, de los acontecimientos actuales extremos en el clima como la presencia de sequias, incendios, inundaciones, tsunamis, huracanes y tifones, entre otros, que continúan acelerando el daño ambiental. Para ayudar

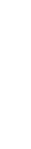


E-ISSN 2322-7028

Vol. 13 No. 2

Jul - Dic 2016

Cúcuta, Colombia en la mejora del medio ambiente, el sector salud se ha visto en la necesidad de crear infraestructuras y programas que den respuesta a emergencias, mediante diferentes formas de proporcionar agua potable y el mejoramiento en la prestación de servicios de salud (7).

Hoy en día, las políticas creadas han permitido preservar o mitigar el daño y velar por la protección y preservación del medio ambiente, implementando buenas prácticas ambientales que minimicen, controlen, eviten y compensen el impacto negativo generado, mediante compromisos como el manejo integral del uso del agua, el manejo integral del uso de la energía, el uso racional del papel, programas de concientización del aseo y el sentido de autodisciplina (26).

También se motiva a la reducción de emisiones (gaseosas, liquidas, sólidas y de ruido), manejo adecuado de vertimientos líquidos, reducción del ruido -cultura del silencio-, manejo integral del componente arbóreo y paisajístico, control integral de plagas y vectores, manejo seguro de productos químicos, compras verdes y cultura de conservación medio ambiental. Como compromiso se han creado estrategias en los diferentes hospitales para amortiguar los daños al ambiente (26).

Dentro de este contexto, se puede indicar que el entorno hospitalario es un lugar peligroso para la salud al generar riesgos biológicos (infecciones nosocomiales, bacterias, virus, protozoarios, toxinas, hongos, alérgenos), físicos (descargas eléctricas, cortes, caídas), no mecánicos (ruido, vibraciones, radiaciones ionizantes y no ionizantes, calor, iluminación, microclima) o mecánicos (lesiones intencionales, no intencionales y autoinflingidas), químicos (ácidos, el formol, los gases de anestesia, las radiaciones, orgánicos e inorgánicos, etc.) $(1,27)$.

De otro lado, existen otros factores en el área hospitalaria que lo hacen un lugar perjudicial para la salud y el ambiente: los agentes peligrosos (explosivos), sustancias inflamables (comburentes), tóxicos (inflamables, corrosivos, irritantes, teratógenos), de riesgos sociales (presentan agresiones y demandas), los riesgos ocasionados a la organización del trabajo y psicosociales (stress, adición a fármacos, desmotivación, tabaquismo, alcoholismo, conductas sexuales riesgosas y violencia), que pueden alterar el estado de salud de los pacientes y la respuesta al manejo de la enfermedad ocasionada por el hospital (27-28).

En este sentido, los elementos que pueden producir contaminación y enfermedades cuando no se manejan adecuadamente son los residuos hospitalarios -sólidos, químicos y tóxicos-. Cuando hay exposición a estas sustancias se pueden generar afecciones graves como asma y cáncer y, además, son fuente importante en la contaminación del aire y generan riesgo, por el uso de productos utilizados en la atención de salud (anatomopatológicos y cortopunzantes), para quienes entren en contacto con ellos (7). En Bogotá se evidencia que, en el año 2013, los centros de salud generaron 49 mil toneladas de residuos hospitalarios (29).

Enlos hospitales también se forman grandes cantidades de residuos comunes como envases, papel, comida, etc., que pueden llegar a representar alrededor del $80 \%$ de los residuos (30). La Organización Mundial de la Salud (OMS) enuncia que un hospital de gran tamaño puede producir hasta una tonelada de residuos por día y los generados por residuos infecciosos lo estiman en un $40 \%$ de los casos de hepatitis y el $12 \%$ de los casos del virus de inmunodeficiencia adquirida (VIH) en el mundo cuando se exponen en el ámbito de trabajo (31-32).

Los riesgos de contaminación hospitalaria se incrementan en los hospitales debido a que son lugares en donde se produce contaminación de diferentes áreas, ya sean internas o externas, tanto para los que se encuentran dentro como para los de afuera. Por ejemplo, las infecciones nosocomiales -aquellas que son adquiridas cuando se ingresa al hospital y que se manifiestan 48 horas después del ingreso del paciente al hospital-, también se incrementan cuando los pacientes llevan una alta hospitalización, adquiriendo los microorganismos del hospital $(28,33)$.

Dentro de las estadísticas se evidencia que un $3 \%$ y $5 \%$ de los pacientes que han ingresado a la institución hospitalaria adquieren una nueva infección, generando un alto costo económico anual, ocasionando complicaciones y hasta la muerte. Entre de las infecciones más frecuentes se evidencia: 15 $\%$ - $20 \%$ neumonía, $25 \%$ la infección de herida quirúrgica, y entre $5 \%$ - $7 \%$ bacteremia asociada a dispositivos intravasculares $(28,34)$. 


\section{El hospital verde}

Como estrategia de salud pública para generar reducción de contaminación hospitalaria se han creado normas y controles para disminuir la contaminación y contribuir al bienestar general del ambiente, las personas y las comunidades. Por esta razón se han organizado los hospitales verdes y saludables, con el objetivo de controlar el uso de los desechos, fomentado prácticas saludables y ecológicas en los hospitales de la ciudad de Bogotá y el país (7).

Hospital verde y saludable se define como "un establecimiento que promueve la salud pública reduciendo continuamente su impacto ambiental y eliminando, en última instancia, su contribución a la carga de morbilidad. Un hospital verde y saludable reconoce la relación que existe entre la salud humana y el medio ambiente y lo demuestra a través de su administración, su estrategia y sus operaciones" $(7,35)$.

El objetivo de la creación de estas medidas es recuperar el medio ambiente e introducir una conciencia para disminuir los impactos negativos sobre éste, organizando acciones de prevención primaria para optimizar la salud ambiental de las comunidades, además de conformar una economía verde y un ambiente sostenible.

Es decir, se crea la necesidad de regular los recursos naturales y la calidad del ambiente; también de controlarlo para así poder garantizar una buena sostenibilidad ambiental y mejorar la calidad de vida (36). Además, desde la Organización de las Naciones Unidas (37), se propone como Séptimo Objetivo del Milenio (ODM) garantizar la sostenibilidad del medio ambiente $\mathrm{y}$, como primera meta "incorporar los principios del desarrollo sostenible en las políticas y los programas nacionales y reducir la pérdida de recursos del medio ambiente" (37), para lo cual el sector salud realiza acciones encaminadas a disminuir este impacto ambiental, cumpliendo las políticas.

En el camino del cambio, los hospitales verdes priorizan cinco aspectos importantes:

- Componente del agua: "orientado al uso racional del agua y la disminución en el consumo y prestación del servicio de salud, implementando buenas prácticas de manejo, tratamiento, reutilización y disposición de la misma". Una llave que gotea desperdicia más de 75 litros de agua por día (2).

- Componente de energía: "pretende disminuir el consumo e implementar tecnologías limpias que reduzcan el uso de energía generada por combustibles fósiles". Un bombillo ahorrador utiliza entre un $50 \% \mathrm{y}$ un $80 \%$ menos de energía que una bombilla normal incandescente para producir la misma cantidad de luz y reducción de gases por efecto invernadero $(2,25)$.

- Componente de residuos: "promueve la gestión integral de los mismos, reduciendo su volumen y toxicidad e implementando prácticas que permitan optimizar su tratamiento y disposición de manera segura" (2). Los prestadores de servicios de salud en la ciudad de Bogotá, en el año 2012, generaron más de 43 millones de kilogramos de residuos, de los cuales 59,66\% son residuos no peligrosos (ordinarios, biodegradables, inertes y reciclables) $(2,25)$.

- Componente áreas verdes: "rescatar al ser humano de los procesos de endurecimiento de su entorno, armonizando su relación con espacios verdes, para lo cual se requieren intervenciones integrales como la agricultura urbana, terrazas, muros y techos verdes que contribuyan al mejoramiento paisajístico" (2).

- Componente de sustancias químicas: "orientado a reducir y reemplazar las sustancias químicas tóxicas como el mercurio, mediante la implementación de tecnologías limpias de productos farmacéuticos y equipos biomédicos e industriales más seguros y eficientes" (2). Los prestadores de servicios de salud en la ciudad de Bogotá, en el año 2012, generaron más de 43 millones de kilogramos de residuos, de los cuales un 40,4 $\%$ fueron residuos peligrosos (biológicos o infecciosos, químicos y administrativos) (2).

En Colombia también se encuentran ejemplos de hospitales verdes:

- En Cali, desde el 2010, se empezaron a ver los beneficios y bondades con la instalación
ISSN 1794-9831

E-ISSN 2322-7028

Vol. 13 No. 2

Jul - Dic 2016

Cúcuta, Colombia 
ISSN 1794-9831

E-ISSN 2322-7028

Vol. 13 No. 2

Jul - Dic 2016

Cúcuta, Colombia de dispositivos para el ahorro de agua y la implementación y digitalización de la unidad de rayos X en un hospital de la localidad. En el año 2012 se realiza el cambio del termómetro de vidrio y los tensiómetros por equipos digitales, disminuyendo la contaminación por metales pesados. En cuanto al almacenamiento de agua, se utilizan tanques que captan el agua de la quebrada El Chambón; para el manejo de la energía eléctrica se utilizan calentadores solares en el área de urgencias, hospitalización y partos, además de paneles solares como fuente de energía alternativa para postes solares con luminaria LED en el alumbrado público $(35,38)$.

- En Bucaramanga se han puesto en ejecución y evaluación los programas de Hospital Verde en el uso eficiente de energía, logrando disminuir el consumo en 3.419.300 kilovatioshora (kwh) en un mes; la gestión integral de residuos sólidos y líquidos se redujo en 26.2 toneladas de residuos peligrosos; el uso eficiente del agua logró disminuir en 72.300 $\mathrm{m} 3$ el consumo mensual, junto con el manejo integral de sustancias químicas. Estos datos se muestran como resultados ambientales obtenidos (40).

- Adicionalmente, se destacaron 10 instituciones con el premio Hospital Verde por buen desempeño ambiental, mostrando una reducción de $19.557 \mathrm{~m} 3$ de agua, el ahorro de energía fue de $994.855 \mathrm{kwh}$ en el año y la reducción de dióxido de carbono fue de 247 toneladas año. Otro hospital de Bucaramanga también recibió premios por aplicación de las normas ambientales y obtuvo reconocimiento de Hospital Verde en la categoría de Mejor Desempeño Ambiental (41).

- En Medellín, un hospital se convirtió en Hospital Verde de Colombia y fue el primero en Latinoamérica en aplicar al certificado LED otorgado en Febrero 16 de 2013 en la categoría plata, distinción que significa liderazgo en diseño energético y ambiental. Además, para el

"LED viene del inglés L.E.D (Light Emitting Diode) emisor de luz. Se trata de un cuerpo semiconductor sólido de gran resistencia que, al recibir una corriente eléctrica de muy baja intensidad, emite luz de forma eficiente y con alto rendimiento (39). manejo del agua se reciclaron las aguas lluvias para los sistemas de riego, el uso de paneles solares y el uso de un sistema que emplea el calor producido por los calentadores de agua para los pacientes. En cuanto a las áreas verdes, el hospital estará rodeado de zonas verdes y contará con jardines de curación, es decir, áreas donde los pacientes podrán tomar el sol y caminar, y la eliminación de cubiertas duras para sustituirlas por cubiertas verdes (42).

- En Boyacá, un hospital recibió reconocimiento de Hospital Verde por segunda vez, por cumplir el objetivo de implementar oportunidades que generan beneficios económicos y ambientales. Ha disminuido en 7.813 metros cúbicos el consumo de agua, en materia energética se consiguió reducir 145 kilowatios hora año y se logró una reducción de 2.858 kilogramos de residuos peligrosos (43).

- En Bogotá, los hospitales también han participado en el programa de hospitales verdes, iniciativa que ha logrado extender buenas prácticas ambientales de los niños a sus padres en el uso de las buenas prácticas de reciclaje para el cuidado del medio ambiente. También se ha motivado a trabajar por lograr un hospital verde, por un mundo sano con educación en el manejo de las huertas urbanas que ha contribuido a mejorar la salud mental y quienes han sido educados en labores agroecológicas como terapia ocupacional (2).

Otro logro alcanzado en los hospitales de Bogotá es la estrategia educativa y la instalación de sanitarios de bajo consumo de agua; entre diciembre de 2013 y febrero de 2014 se logró una disminución del 29 $\%$ (435 m3 menos) en el consumo de agua, lo que representa un ahorro económico del $41 \%(2,44)$. En cuanto al manejo del área de rayos $\mathrm{X}$, se eliminó el uso de líquidos reveladores y fijadores y ahora los usuarios pueden obtener las radiografías en disco compacto $(\mathrm{CD})$ o físico y las imágenes diagnosticas de más complejidad pueden ser consultadas por las redes internas del hospital $(12,44)$.

Otras medidas implementadas fueron: el uso de amalgamas en lugar de resinas en los procedimientos odontológicos, alcanzando un $62 \%$ en el primer trimestre del 2014, y la eliminación del uso de 
termómetros de mercurio e implementación del termómetro digital. En cuanto al manejo de la energía, se disminuyó el consumo de gas con la implementación de tecnología de panel solar para el calentador. También se produjeron baterías ecológicas sanitarias que utilizan sólo un litro por descarga, reduciendo a 450 litros/día de agua. Además, se instaló tecnología LED, ahorrando el $60 \%$ de consumo de energía y ahorro diario de 1.080 litros de agua potable por la instalación de sanitarios de bajo consumo de agua y orinal seco $(2,12,44-45)$.

En relación con el manejo de los residuos hospitalarios, en la red de hospitales hubo disminución en un 10.32 $\%$, y el sector de Chapinero fue el que obtuvo mayor disminución en generación de residuos con 51,75 $\%$. También se logró un aumento en el volumen de residuos reciclados (45).

A esto se añade que los sistemas de salud y los hospitales pueden aumentar su posición económica ya que gozan de buena reputación en las comunidades, para así poder contribuir a alcanzar los Objetivos de Desarrollo del Milenio relacionados con la salud y la sustentabilidad, para impulsar una economía verde y sustentable; además, a través de los lineamientos y estrategias de La Agenda Global para Hospitales Verdes y Saludables, se plantean las terrazas ecológicas o verdes como alternativas para disminuir las moles de cemento y concentrar de nuevo la vegetación, generar espacios de relajación y volver a embellecer las zonas. Estas también se conocen como los techos vegetales, techos verdes, terrazas vegetales, techos ecológicos, terrazas verdes, techos vivos, cubiertas verdes o cubiertas vegetales (46-47).

Para contrarrestar el daño, el sector salud promueve la implementación de las terrazas verdes como medidas de innovación que pueden mejorar el medio ambiente y el entorno de las zonas urbanas, la relación entre la ciudad y la naturaleza. Las terrazas verdes son definidas como "cubiertas de plantas que se construyen en las terrazas de los edificios y casas, que colaboran en reconstruir el lazo entre la naturaleza y las personas" (12) y, además, para regular la temperatura (o aislamiento térmico) entre los edificios y la calidad de los espacios.

Molina (9) afirma que desde el horizonte ambiental las áreas verdes cumplen la función de pulmón de edificios de la ciudad, en conjunto con los arboles del área pública y los jardines y zonas privadas, pero con la contaminación que existe en la atmosfera de Buenos Aires, todas esas áreas verdes no resultan suficientes para que mejore el aire y que sea de buena calidad.

Como beneficios de los techos o cubiertas verdes se puede identificar el control de la contaminación por dióxido de carbono (CO2), polvo, ruido o smog, que amortigua el efecto del calor urbano y absorbe hasta un $80 \%$ de la radiación solar, demorando la llegada del agua lluvia al alcantarillado y mitigando las inundaciones a partir de un efecto conocido como esponja en las plantas y la tierra, ayudando en muchas construcciones mediante un sistema de reaprovechamiento del agua y aumento de las zonas verdes de una ciudad, transformando el paisaje urbano. También se generan áreas de relajación y espacios agradables de trabajo, aparte de la mejora estética y valorización de la propiedad $(9,48)$.

\section{Políticas ambientales}

Para reducir la huella ambiental, el gobierno y el sector salud sugieren mejorar la estructura de los edificios, fomentar los planes de viaje verdes, la gestión y reducción de los desechos, el aprovechamiento de las fuentes de energía renovables (49-50) y así impulsar las superficies verdes, la oxigenación natural y permitir la generación de una cultura ambiental para reconstruir la relación entre las plantas y las personas. Los espacios verdes y/o áreas abiertas cubiertas con vegetación benefician a la ciudad y a sus habitantes, ya que por medio de ellos se controla la temperatura, se absorbe el agua de lluvia, se desacelera el escurrimiento, se fomenta la biodiversidad y se enfrenta el daño ambiental y a la salud de las personas, contribuyendo a la solución de la problemática generada por el cambio climático.

En cuanto al saneamiento ambiental, el estado colombiano a través del Ministerio de Salud ha promulgado la Ley 09 de 1979 (51), dando inicio a la era del derecho sanitario y estableciendo el componente jurídico en tres áreas: saneamiento ambiental, atención a las personas y vigilancia y control sanitarios. Al mismo tiempo, para los residuos sólidos, el Ministerio de Salud a través de la Dirección de Saneamiento Ambiental, estableció un programa nacional de aseo urbano y también formuló un programa nacional de reciclaje, en el año 1983, como estrategia para disminuir las cantidades de basura y regular su manejo (51-52).
E-ISSN 2322-7028

Vol. 13 No. 2

Jul - Dic 2016

Cúcuta, Colombia 
E-ISSN 2322-7028

Vol. 13 No. 2

Jul - Dic 2016

Cúcuta, Colombia
Asimismo, se inició el desarrollo de un programa de rellenos sanitarios manuales para municipios menores de 25.000 habitantes $(90 \%$ del total de municipios del país). El éxito alcanzado por el Ministerio de Salud y Protección Social fue escaso en el direccionamiento del programa de aseo urbano, debido a la poca importancia que, en general, los diferentes gobiernos le han asignado al sector de residuos sólidos y la salud ambiental (52).

Los problemas ocasionados a la salud ambiental exigen y plantean a los sistemas de salud un mayor recurso económico, puesto que los efectos generados por el cambio climático, la contaminación por residuos y el mal manejo de los recursos hacen de las ciudades lugares hostiles para la mayoría de las plantas, animales y personas, aumentando la incidencia de enfermedades.

Para superar estos efectos se han creado los techos verdes, que hoy en día representan un hábitat saludable en el paisaje urbano. Debido a que han crecido los factores que aumentan los niveles de ruido y de estrés que agravan la calidad de vida de los residentes del área urbana, este fenómeno se logra reducir con la creación de espacios verdes de recreación y relajación, tal como lo afirma la Red Global de Hospitales Verdes y Saludables (7): "Varios estudios han relacionado los efectos calmantes de las plantas con tiempos más cortos de recuperación de pacientes enfermos".

Como ya se había mencionado anteriormente, los hospitales se consideran lugares peligrosos para la salud porque generan riesgos biológicos, físicos, peligrosos, tóxicos, riesgos sociales, riesgos ocasionados por la organización del trabajo y riesgos psicosociales que resultan perjudiciales para la salud y el ambiente. Se indica que en los hospitales de países en desarrollo todos los residuos que se generan se mezclan y se queman en incineradores de baja tecnología y alto grado de contaminación o bien a cielo abierto, sin ningún tipo de control, evidenciando que la incineración de residuos hospitalarios genera grandes cantidades de dioxinas, mercurio y otras sustancias contaminantes (52).

Sin embargo, los hospitales y los sistemas de salud de todo el mundo se han adaptado al flagelo del cambio climático y, de igual manera, se han propuesto promover la sustentabilidad, la salud ambiental y la organización de una mayor equidad sanitaria mediante la inversión saludable, las compras verdes y la implementación de operaciones sustentables para potenciar la posición económica y la reputación moral, con el fin de alcanzar los Objetivos de Desarrollo del Milenio relacionados con la salud y la sustentabilidad, además de impulsar, al mismo tiempo, una economía verde (7).

Países como España, EE. UU., algunos de Europa y otros de América Latina cuentan con hospitales sustentables, ecológicos y seguros dentro de su infraestructura. En vía de desarrollo se encuentra el hospital de México, pero todos tienen el compromiso de implementar medidas desde el sector salud, gobierno, personal médico, ingenieros, administrativos y usuarios para alcanzar fácil, rápida y eficazmente el compromiso de disminuir el daño ambiental (25).

El personal médico, y especialmente enfermería, cumplen una función importante que es educar y dar a conocer la importancia de las nuevas políticas ambientales para lograr así su implementación y ofrecer servicios innovadores con el ambiente y promocionar ambientes saludables. Igualmente, al convertirse en un miembro de un hospital equipo verde $^{\dagger}(53)$, el personal de enfermería también puede promover la salud ambiental interviniendo en la selección de productos y la eliminación de aquellos que no sean apropiados, contribuyendo a la preservación del medio ambiente y de la salud.

\section{CONCLUSIONES}

Es importante que el profesional de enfermería, desde la formación universitaria, conozca la temática ambiental y los programas de hospitales verdes para generar una cultura ambiental y de entornos saludables, teniendo en cuenta el cuidado que debe implementarse con los nuevos modelos de salud familiar y comunitaria, que pretenden impulsar los objetivos del Milenio y los objetivos del Desarrollo Sostenible.

El personal de salud, en especial los profesionales de enfermería, cumple una función importante, a través de la educación, para la aplicación e implementación de las nuevas políticas ambientales que permitan establecer los

\footnotetext{
ॠ Un equipo verde es un grupo multidisciplinario de trabajadores del hospital que apoyan e informan a otros miembros del personal sobre las prácticas institucionales sostenibles con el medio ambiente (53).
} 
servicios amigables e innovadores con el ambiente, en cada uno de los espacios donde se realice su trabajo.

Se pretende mejorar el medio ambiente con estrategias conjuntas, mediante el fortalecimiento del desarrollo local, integral y sostenible de los países, municipios y ciudades, de todas las instituciones y la ciudadanía, para continuar fomentando la protección del medio ambiente como determinante de la salud en la población y así disminuir la morbimortalidad generada por la contaminación.

Modificar las costumbres en el manejo de los residuos es relevante para disminuir los efectos causados al medio ambiente y que ocasionan escasez de agua potable, evitando que continúe el cambio climático y previniendo las enfermedades generadas por la contaminación.

Es importante resaltar que los hospitales han adoptado políticas para generar responsabilidad social, creando acciones para mejorar el ambiente y atenuar los impactos negativos que se han generado con la prestación de los servicios de salud, promoviendo el programa de hospitales verdes para lograr reducir el impacto ambiental y los índices de morbilidad y mortalidad.

La estrategia de las terrazas verdes ayuda a crear conciencia en cuanto a la ecología y el fortalecimiento del vínculo que debe existir entre la naturaleza y la ciudad, para disminuir el calentamiento de la ciudad, reducir el smog, ejercer el efecto esponja entre la tierra y las plantas, mejorar la calidad de vida de la población, optimizar y evaluar la salud de los ecosistemas y preservar la biodiversidad.

A través de esta revisión bibliográfica se describió la importancia de la estrategia de las terrazas verdes, la cual contribuye a mejorar los recursos económicos $\mathrm{y}$ ambientales de las instituciones para poder ser socialmente sustentables, promocionar un ahorro financiero y continuar promulgando el cuidado del medio ambiente y la salud humana, siendo parte importante los entes gubernamentales, la sociedad y el equipo de salud, quienes podrán diseñar y aplicar medidas para enfrentar estos efectos en las áreas rurales, urbanas y en la población en general.

\section{CONFLICTO DE INTERESES}

La autora declara no tener ningún conflicto de intereses. 


\section{REFERENCIAS BIBLIOGRÁFICAS}

Jul - Dic 2016

Cúcuta, Colombia

1. Albert LA. Capítulo 4. Contaminación Ambiental origen, clases, fuentes y efectos. En: Albert LA, Jacott M. México tóxico. México: Siglo XXI Editores; 2015.

2. Alcaldía Mayor de Bogotá D.C. Hospitales Verdes, Bogotá por un Mundo Sano. Boletín Virtual No. 1 [Internet]. 2014 [consultado 28 de junio de 2016]. Disponible en: http://www.esevictoria.gov.co/joomla31/images/stories/ ambiental/Boletin1_Hospitales_Verdes.pdf

3. Organización de las Naciones Unidas (ONU). Objetivos de Desarrollo Sostenible. 17 Objetivos para Transformar Nuestro Mundo. [Internet]. 2015 [consultado 27 de junio de 2016]. Disponible en: http://www.un.org/ sustainabledevelopment/es/objetivos-de-desarrollo-sostenible/

4. Aguilar L. Contaminación Ambiental. Qué es la Contaminación Ambiental. [Internet]. 2009 [consultado 27 de junio de 2016]. Disponible en: http://contaminacion-ambiente.blogspot.com.co/2006/10/que-es-lacontaminacion-ambiental.html

5. Alcaldía Mayor de Bogotá D.C. Hospitales Verdes, Bogotá por un Mundo Sano. Boletín Virtual No. 3 [Internet]. 2015 [consultado 11 de marzo de 2016]. Disponible en: https://issuu.com/75632/docs/boletin_hv_03

6. Peñalosa E. Programa de Gobierno Coalición Equipo por Bogotá - Cambio Radical: Recuperemos Bogotá. [Internet]. 2015 [consultado 23 de marzo de 2016]. Disponible en: http://www.colombia.com/elecciones/2015/ regionales/images/plan-de-pe\%C3\%B1alosa.pdf

7. Red Global de Hospitales Verdes y Saludables. Agenda Global para Hospitales Verdes y Saludables. Un marco integral de salud ambiental para los hospitales y los sistemas de salud de todo el mundo. [Internet]. 2011 [consultado 30 de julio de 2016]. Disponible en: http://www.hospitalesporlasaludambiental.net/wp-content/ uploads/2011/10/Agenda-Global-para-Hospitales-Verdes-y-Saludables.pdf

8. Paramo P. La Investigación en Ciencias Sociales: Estrategia de Investigación. $1^{\mathrm{a}}$ ed. Bogotá D.C.: Universidad Piloto de Colombia, Gráficas Gilpor Ltda.; 2011.

9. Molina-Dato DL. Terrazas Verdes, Diseño y Sustentabilidad. [Tesis en internet]. Argentina: Universidad de Palermo; 2013 [consultado 20 de julio de 2016]. Disponible en: http://fido.palermo.edu/servicios_dyc/ proyectograduacion/archivos/2722.pdf

10. República de Colombia, Ministerio de Salud y Protección Social. Salud Ambiental. [Internet]. 2016 [consultado 20 de julio de 2016]. Disponible en: https://www.minsalud.gov.co/salud/Paginas/Salud-ambiental.aspx

11. Alcaldía Mayor de Bogotá D. C. Política Distrital de Salud Ambiental para Bogotá D. C. 2011 - 2023. [Internet]. Bogotá D. C.: Alcaldía Mayor de Bogotá D.C.; 2011 [consultado 8 de junio de 2016]. Disponible en: http://ambientebogota.gov.co/c/document_library/get_file?uuid=5572b766-73ed-4087-bf27$21 \mathrm{fc} 6406 \mathrm{a} 810 \&$ groupId $=55886$

12. Organización Mundial de la Salud (OMS). Salud Ambiental. [Internet]. 2016 [consultado 6 de junio de 2016]. Disponible en: http://www.who.int/topics/environmental_health/es/

13. Organización Mundial de la Salud (OMS). Ambientes Saludables y Prevención de Enfermedades. Hacia una estimación de la carga de morbilidad atribuible al medio ambiente. [Internet]. 2016 [consultado 6 de junio de 2016]. Disponible en: http://www.who.int/quantifying_ehimpacts/publications/prevdisexecsumsp.pdf

14. República de Colombia, Superintendencia de Servicios Públicos Domiciliarios (SSPD). Situación de la disposición final de residuos sólidos en Colombia - Diagnostico 2011. [Internet]. Colombia: SSPD: 2011 [consultado 7 de junio de 2016]. Disponible en: http://www.superservicios.gov.co/content/download/901/13765

15. Hoornweg D, Bhada-Tatha P. What a Waste. A Global Review of Solid Waste Management. Urban Development Series Knowledge Papers. No. 15. [Internet]. Washington, DC: World Bank; 2012 [consultado 6 de junio de 2016]. Disponible en: http://siteresources.worldbank.org/INTURBANDEVELOPMENT/ Resources/336387-1334852610766/What_a_Waste2012_Final.pdf

16. Alcaldía de Envigado, Secretaria del Medio Ambiente y Desarrollo Rural. Guía para el adecuado manejo de los residuos sólidos y peligrosos. [Internet]. 2011 [consultado 28 de junio de 2016]; 32 p. Disponible en: http:// www.envigado.gov.co/Secretarias/SecretariadeMedioAmbienteyDesarrolloRural/documentos/publicaciones/ Guia_residuos.pdf

17. Rodríguez SC. Residuos Sólidos en Colombia: su manejo es un compromiso de todos. L' esprit ingènieux [Internet]. 2011 [consultado 7 de junio de 2016]; 2(11). Disponible en: http://revistas.ustatunja.edu.co/index. php/lingenieux/article/view/117/92

18. Organización Panamericana de la Salud (OPS), Organización Mundial de la Salud (OMS). Informe de Seguimiento Gestión de Residuos Sólidos en Colombia. Salud ambiental y entornos saludables.[Internet]. 2003 [consultado 5 de junio de 2016]. Disponible en: http://www.paho.org/col/index.php?option=com_content\&view 
$=$ article\&id=836:informe-de-seguimiento-gestion-de-residuos-solidos-en-colombia\&Itemid=361

19. Contreras C. Manejo Integral de Aspectos Ambientales - residuos sólidos. Pontificia Universidad Javeriana. [Internet]. 2006 [consultado 7 de junio de 2016]. Disponible en: http://www.javeriana.edu.co/ier/recursos_user/ IER/documentos/OTROS/Pres_Residuos_CamiloC.pdf

20. Organismo Provincial para el Desarrollo Sostenible (OPDS), Subsecretaria de Educación. Gestión integral de Residuos Sólidos Urbanos. Documento destinado a docentes. [Internet]. Buenos Aires; OPDS; 2010 [consultado 8 de junio de 2016]. Disponible en: http://www.opds.gba.gov.ar/uploaded/File/residuos_03_10.pdf

21. Organización Panamericana de la Salud (OPS), Asociación Interamericana de Ingeniería Sanitara Y Ambiental (AIDIS), Banco Interamericano de Desarrollo (BID). Informe de la Evaluación Regional del Manejo de Residuos Sólidos Urbanos en América Latina y el Caribe 2010. [Internet]. OPS/AIDIS/BID; 2010 [consultado 8 de junio de 2016]. Disponible en: http://idbdocs.iadb.org/wsdocs/getdocument.aspx?docnum=36466973Contreras C.

22. Manejo Integral de Aspectos Ambientales - residuos sólidos. Pontificia Universidad Javeriana. [Internet]. 2006 [consultado 7 de junio de 2016]. Disponible en: http://www.javeriana.edu.co/ier/recursos_user/IER/documentos/ OTROS/Pres_Residuos_CamiloC.pdf

23. Díaz E. El mal manejo de la basura y la proliferación de las enfermedades. Fundación Gilberto Montero. [Internet]. República Dominicana; 2012 [consultado 23 de julio de 2016]. Disponible en: http://www.gilbertomontero.com/ tireo/el-mal-manejo-de-la-basura-y-la-proliferacion-de-las-enfermedades/

24. Bustos-Flores C. La problemática de los desechos sólidos. Economía [Internet] 2009 [consultado 23 de julio de 2016]; XXXIV (27): 121-144. Disponible en: http://www.saber.ula.ve/bitstream/123456789/30305/1/articulo5. pdf

25. Red Global de Hospitales Verdes y Sustentables. Informe Anual de Progreso 2014. [Internet]. 2014 [consultado 8 de junio de 2016]. Disponible en: http:/hospitalesporlasaludambiental.net/wp-content/uploads/2015/04/ Informe-anual-Red-Global-2014.pdf

26. República de Colombia, ESE Hospital del Sur Itagüí "Gabriel Jaramillo Piedrahita”. Política Manejo Ambiental. [Internet]. 2014 [consultado 8 de junio de 2016]. Disponible en: http://www.hospitaldelsur.gov.co/politicasinstitucionales/114-politica-de-manejo-ambientalado 23 de julio de 2016]. Disponible en: http://www.saber.ula. ve/bitstream/123456789/30305/1/articulo5.pdf

27. Alcaldía Mayor de Bogotá D.C. Vigilancia en Salud Pública. Gestión Integral de Residuos Hospitalarios y Similares. [Internet]. 2012 [consultado 8 de junio de 2016]. Disponible en: http://www.saludcapital.gov.co/ sitios/VigilanciaSaludPublica/Paginas/ResiduosHospitalarios.as

28. Romero M, Álvarez M, Álvarez A. Los factores ambientales como determinantes del estado de salud de la población. Rev Cubana Hig Epidemiol [Internet]. 2007 [consultado 8 de junio de 2016]; 45(2). Disponible en: http://www.redalyc.org/pdf/2232/223219929001.pdf

29. www.equilibriummedicinanatural.com. Llegaron los "Hospitales Verdes" a Bogotá. Equilibrium [Internet]. 2014 [consultado 28 de junio de 2016]. Disponible en: http://www.equilibriummedicinanatural.com/llegaronlos-hospitales-verdes-a-bogota/

30. Hospital Sagrado Corazón E.S.E. Departamental Norcasia - Caldas. Plan de Gestión Integral de Residuos Hospitalarios y Similares. [Internet]. Norcasia; Hospital Departamental Sagrado Corazón; 2012 [consultado 8 de junio de 2016]. Disponible en: http://esehospitalsagradocorazon-norcasia-caldas.gov.co/apc-aa-fil es/65306538333232303631336663626361/pgirh.pdf

31. TN Relaciones. Contaminación Hospitalaria. [Internet] 2016 [consultado 11 de marzo de 2016]. Disponible en: http://www.tnrelaciones.com/cm/preguntas_y_respuestas/content/206/3289/es/contaminaci\%F3n-hospitalaria. html

32. Quinto Y, Jaramillo LM, Cardona JA. Conocimientos y prácticas de los trabajadores de un hospital sobre el manejo de residuos hospitalarios, Chocó, Colombia, 2012. MéD. UIS. [Internet]. 2013 [consultado 8 de junio de 2016]; 26(1): 9-20. Disponible en: http://www.scielo.org.co/pdf/muis/v26n1/v26n1a02.pdf

33. Sandoval-Flores L. Aspectos físicos, químicos y biológicos de la contaminación hospitalaria. Revista de Medicina e Investigación [Internet]. 2014 [consultado 8 de junio de 2016]; 2(1):35-41. Disponible en: http://apps.elsevier. es/watermark/ctl_servlet?_f $=10 \&$ pident_articulo $=90278770 \&$ pident_usuario $=0 \&$ pcontactid $=\&$ pident_revista $=$ $353 \&$ ty $=4 \&$ accion $=L \&$ origen $=$ zonadelectura $\& w e b=w w w . e l s e v i e r . e s \& l a n=e s \&$ fichero $=353 \mathrm{v} 02 \mathrm{n} 01 \mathrm{a} 90278770 \mathrm{p}$ df001.pdf

34. Organización Mundial de la Salud (OMS). Prevención de las Enfermedades Nosocomiales. Guía Práctica. [Internet]. 2005 [consultado 24 de julio de 2016]. Disponible en: http://www.who.int/csr/resources/publications/ ES_WHO_CDS_CSR_EPH_2002_12.pdf

35. Red de Salud de Ladera ESE. Hospital de Cañaveralejo. Verde y Saludable. [Internet]. 2014 [consultado 8 
de junio de 2016]. Disponible en: http://www.saludladera.gov.co/index.php/component/phocadownload/ category/36-anexos-hospital-verde

36. Hernández JC. Caracterización de la gestión de residuos hospitalarios y similares en CAMI Vista Hermosa, Bogotá. Respuestas [Internet]. 2016 [consultado 8 de junio de 2016]; 21(1): 6-15. Disponible en: http://revistas. ufps.edu.co/ojs/index.php/respuestas/article/view/630/634

37. Organización de las Naciones Unidas (ONU). Objetivos del Desarrollo del Milenio y más allá del 2015. Objetivo 7: Garantizar la sostenibilidad del medio ambiente. [Internet]. 2015 [consultado 8 de junio de 2016]. Disponible en: http://www.un.org/es/millenniumgoals/environ.shtml

38. Unidad de Apoyo Gestión de Comunicaciones. Cali con el Único Hospital Verde y Saludable del País. Red de Salud de Ladera. [Internet]. 2014 [consultado 9 de junio de 2016]. Disponible en: http://www.cali.gov.co/ publicaciones.php?id=103382\&dPrint $=1$

39. Que es Luz LED. Tecnología y Educación. [Internet]. 2012 [consultado 28 de junio de 2016]. Disponible en: http://www.tecnologiayeducacion.com/\%C2\%BFque-es-luz-led/

40. Hernández TC. Implementación del Programa Hospital Verde en el Instituto del Corazón en Bucaramanga. [Monografía en internet]. Bucaramanga: Universidad Industrial de Santander; 2010. [consultado 9 de junio de 2016]. Disponible en: http://repositorio.uis.edu.co/jspui/bitstream/123456789/7499/2/136495.pdf

41. Empresas de salud del sector reconocidas con el galardón 'Hospital Verde'. Revista Gente de Cañaveral. [Internet]. 2012 [consultado 9 de junio de 2016]. Disponible en: http://www.gentedecanaveral.com/2012/06/ empresas-de-salud-del-sector-reconocidas-con-el-galardon-hospital-verde/

42. Uribe DF, Arboleda AF. Centros Especializados de San Vicente Fundación; hospital verde con certificación LEED. Revista Ingeniería Biomédica [Internet]. 2015 [consultado 28 de junio de 2016]; 9(18): 51-56. Disponible en: http://www.scielo.org.co/pdf/rinbi/v9n18/v9n18a19.pdf

43. E.S.E. Hospital Regional de Duitama. Certificación como Hospital Verde. [Internet]. 2011 [consultado 28 de junio de 2016]. Disponible en: http://www.hrd.gov.co/documentos/noticias/CertificacionHospitalVerde.pdf

44. Terrazas Verdes en la Universidad Autónoma de Manizales. autonoma.edu.co [Internet]. 2014 [consultado 9 de junio de 2016]. Disponible en: http://www.autonoma.edu.co/attachments/article/650/Terrazas\%20Verdes\%20 en $\% 201 \mathrm{a} \% 20$ Universidad $\% 20$ Aut $\%$ C3\%B3noma\%20de\%20Manizales.pdf

45. Jaimes K. El Distrito entregó balance del programa de Hospitales Verdes. Su vida -Edimédicas. [Internet]. 2014 [consultado 9 de junio de 2016]. Disponible en: http://su-vida.com/node/2367

46. Euclid Chemical Toxement. Techos Vegetales. Un aporte más de Euclid Chemical Toxement al desarrollo sostenible del país. [Internet]. 2013 [consultado 28 de junio de 2016]. Disponible en: http://www.toxement.com. co/brochure/Brochure_Cubiertas_Vegetales_2013.pdf

47. Defensoría del Pueblo de la Ciudad Autónoma de Buenos Aires. Terrazas verdes: innovación urbana y ambiental para mejorar la relación sociedad naturaleza y la calidad de vida de la población en la Ciudad. Área de Ambiente y Desarrollo Sostenible. [Internet]. 2010 [consultado 28 de junio de 2016]. Disponible en: https://9fisicaolaya. files.wordpress.com/2010/11/informe-sobre-terrazas-verdes.pdf

48. Hospital de Suba II Nivel ESE. Eje 3. Compromiso con el Medio Ambiente y Disminución de la Huella Ecológica. [Internet]. 2015 [consultado 9 de junio de 2016]. Disponible en: http://200.93.163.76:8080/moodledata9/137/ moddata/assignment/34/4674/EJE_3._COMPROMISO_CON_EL_MEDIO_AMBIENTE_Y_ DISMINUCION_DE_LA_HUELLA_ECOLOGICA.pdf

49. Organización Mundial de la Salud (OMS). Anexo 2. Reducción de la Huella de Carbono del Sector de la Salud. En: OMS. Proteger la salud frente al cambio climático. Día Mundial de la Salud 2008. [Internet]. 2008 [consultado 28 de junio de 2016]. Disponible en: http://www.who.int/world-health-day/toolkit/annexe\%202-S.pdf

50. República de Colombia, Ministerio de Ambiente y Desarrollo Sostenible. Criterios ambientales para el diseño y construcción de viviendaurbana. [Internet]. Colombia: MinisteriodeAmbientey Desarrollo Sostenible; 2012 [consultado 9 de junio de 2016]. Disponible en: https://www.minambiente.gov.co/images/AsuntosambientalesySectorialyUrbana/ pdf/Sello_ambiental_colombiano/cartilla_criterios_amb_diseno_construc.pdf

51. Congreso de Colombia. Ley 9 de 1979, enero 24, por la cual se dictan Medidas Sanitarias. [Internet]. Bogotá D.E.: Gobierno Nacional; 1979 [consultado 28 de junio de 2016]. Disponible en: http://www.redlactea.org/wpcontent/uploads/decretos/L9.pdf

52. Sánchez G. Desarrollo y medio ambiente: una mirada a Colombia. 2002. Economía y Desarrollo. [Internet]. 2002 [consultado 9 de junio de 2016]; 1(1):79-98. Disponible en: http://www.fuac.edu.co/revista/M/seis.pdf

53. Dermott RM. The Nurse's Role on Green Teams: An Environmental Health Opportunity. Pa Nurse. [Internet]. 2011 [consultado 28 de junio de 2016]; 66(1): 17-21. Disponible en: https:/www1.villanova.edu/content/dam/ villanova/sustainability/McD-LNurseGreenTeam.pdf 